This is the accepted manuscript of the article, which has been published in Attachment \& Human Development. https://doi.org/10.1080/14616734.2018.1530684

\title{
Attachment Security and Cortical Responses to Fearful Faces in Infants
}

\author{
Mikko J. Peltola ${ }^{\mathrm{a}}$, Marinus H. van IJzendoorn ${ }^{\mathrm{b}, \mathrm{c}}$, \& Santeri Yrttiaho $^{\mathrm{d}}$
}

${ }^{a}$ Human Information Processing Laboratory, Psychology, Faculty of Social Sciences, University of Tampere, Finland

${ }^{\mathrm{b}}$ Department of Psychology, Education and Child Studies, Erasmus University Rotterdam, The Netherlands

${ }^{c}$ School of Clinical Medicine, University of Cambridge, UK

${ }^{\mathrm{d}}$ Infant Cognition Laboratory, Faculty of Medicine and Life Sciences, University of Tampere,

Finland

CORRESPONDING AUTHOR: Mikko Peltola, Human Information Processing Laboratory, Psychology, Faculty of Social Sciences, 33014 University of Tampere, Finland. Tel.

+358503186120. E-mail: mikko.peltola@staff.uta.fi

Twitter: @mikkojpeltola

ORCiD ID: Mikko Peltola: 0000-0001-9993-9963; Santeri Yrttiaho: 0000-0002-5724-1222;

Marinus van IJzendoorn: 0000-0003-1144-454X

AUTHOR NOTE: We would like to thank Jukka Leppänen for his contribution to all aspects of this study. This work was supported by grants from the Kone Foundation and the Academy of Finland (\#275519 and \#307657) to MJP, and the Netherlands Organization for Scientific Research (SPINOZA prize) to MHvIJ. 


\begin{abstract}
The present study measured event-related potentials (ERPs) to investigate whether cortical responses to facial expressions of fear are associated with the development of secure and insecure patterns of infant-mother attachment during the first year. Based on previous findings showing reduced attentional biases to fearful faces in infants with insecure and disorganized attachment, we hypothesized that insecure and disorganized attachment would be associated with reduced ERP differentiation of fearful from non-fearful faces. ERPs to facial expressions were measured at 7 months of age and attachment was assessed at 14 months of age with the Strange Situation Procedure $(n=61)$. Occipitotemporal face-sensitive ERP responses particularly in the time range of the N290 component were related to attachment security at 14 months. Only securely attached infants showed age-typical cortical discrimination of fearful from non-fearful faces at 7 months, whereas a similar pattern of ERP responses was not observed in infants with insecure and disorganized attachment. These results add to previous findings by suggesting that patterns of secure and insecure infant attachment are related to early-emerging differences in the perceptual processing of facial emotions, which could have implications for the development of social competence.
\end{abstract}

Keywords: Attachment; Event-related potentials; Facial expressions; Infants 


\section{Introduction}

The first year of life is critical for the development of infants' attachment relationships with caregivers. Secure, insecure, and disorganized patterns of attachment are expressed in infants' behaviors, emotional reactions, and communicative signals when infants separated from the caregiver or experiencing other events triggering feelings of fear and stress, are reunited with their attachment figure (Ainsworth, Blehar, Waters, \& Wall, 1978). Research on the early antecedents of attachment has primarily investigated the factors related to caregiving that are associated with different patterns of attachment formation in infancy. While evidence for the role of sensitive and responsive caregiving on the development of secure attachment has been established, the effect size of the association between parental sensitivity and infant attachment security tends to be modest (De Wolff \& van IJzendoorn, 1997; Verhage et al., 2016). Considerably less is known about infant characteristics predicting developmental differences in attachment patterns. This paper represents an emerging line of research aiming to investigate whether infants' neural and behavioral responses to facial emotion signals are associated with the development of attachment.

Prior to language onset, infants are highly reliant on the caregivers' ability to perceive, interpret, and respond to their communicative signals, and facial expressions form a key channel for transmitting important signals related to attachment within caregiver-infant interaction (cf. Beebe et al., 2010). Facial expressions convey information about the caregiver's emotional state toward the infant, such as signals of affiliation/joy, rejection/anger, fear, sadness, or blunted affect. Caregivers also use facial expressions to mirror infant emotional states, which is essential for the infant's developing differentiation, understanding, and regulation of emotions (Kim et al., 2014; Rayson, Bonaiuto, Ferrari, \& Murray, 2017). As attachment can be reliably assessed with observational methods only after the infant has achieved mobility (Ainsworth et al., 1978), the study of infants' attentional and neural responses to facial emotional information during the first year may reveal early infant-related markers of attachment formation. 
Recently, we (Peltola, Forssman, Puura, van IJzendoorn, \& Leppänen, 2015) began to investigate this question by assessing infants' attention to facial expressions at 7 months in an eyetracking paradigm and their attachment to the mother at 14 months of age with the Strange Situation Procedure (Ainsworth et al., 1978). Given that variations in attachment security are associated with physiological, behavioral, and representational responses to cues signaling threat (Cassidy, Ehrlich, \& Sherman, 2013), we were particularly interested in infants' attentional biases to threat-related facial cues and their relation to attachment development. Fearful and non-fearful (i.e., happy, neutral) facial expressions were presented concurrently with peripheral distractor stimuli, and the difference in the likelihood of performing saccades to the peripheral stimuli from different facial expressions served as an index of attentional bias. Infants who were later classified as securely attached to their mother displayed a robust and age-typical (Forssman et al., 2014; Peltola, Hietanen, Forssman, \& Leppänen, 2013; Peltola, Leppänen, Palokangas, \& Hietanen, 2008) attentional bias to fearful faces, indicated by a reduced likelihood of saccades to peripheral distractor stimuli when attention was engaged on centrally presented fearful faces. This bias was markedly reduced in infants later classified as insecurely attached (i.e., infants with avoidant, resistant, or disorganized attachment classification), and the reduction in attentional bias appeared to be particularly related to increasing signs of attachment disorganization. These results suggest attention to negative emotions as a potential marker of infants' attachment security during the first year.

Complementing the measurements of eye-tracking based attentional biases to faces, recording of brain responses with electroencephalography (EEG) could provide valuable data on the earliest phases of perceptual and attentional processing of faces. Specifically, by measuring eventrelated potential (ERP) components that are related to perceptual sensitivity to variations in facial information as well as ERP components that are more closely associated with variations in attention allocation to faces, we may increase understanding about which phases of facial information 
processing are most sensitive to attachment-related differences in infants. Research investigating the ontogeny of emotional face processing in infancy has shown that by 5 to 7 months of age, ERP components associated with perceptual sensitivity to faces (occipitotemporal N290 and P400 components) and attentional engagement (frontocentral Nc component) begin to reliably differ between fearful and non-fearful facial emotions (Hoehl \& Striano, 2010; Leppänen, Moulson, Vogel-farley, \& Nelson, 2007; Nelson \& de Haan, 1996; Peltola, Leppänen, Mäki, \& Hietanen, 2009; Yrttiaho, Forssman, Kaatiala, \& Leppänen, 2014). Yrttiaho and colleagues (2014) showed that the differential neural responses to fearful vs. non-fearful faces at the latency of the N290 component became more robust from 5 to 7 months of age. The N290 is observed as a negative waveform at around 250-350 ms on occipitotemporal electrodes and considered as an infant precursor of the adult face-sensitive N170 response (de Haan, Johnson, \& Halit, 2003), with its cortical generators likely located in the fusiform gyrus and the superior temporal sulcus (Guy, Zieber, \& Richards, 2016; Sadeh, Podlipsky, Zhdanov, \& Yovel, 2010). Similarly, Peltola et al. (2009) found that the Nc component, which appears as a negative waveform at around 300-600 ms and reflects prefrontal cortical activity related to attentional engagement (Guy et al., 2016; Reynolds \& Richards, 2005), was modulated by fearful expressions at 7 months but not at 5 months of age.

Although ERPs provide useful markers of cortical discrimination of facial emotions during infancy, thus far no studies have investigated potential attachment-related differences in the neural processing of facial emotions at the early stages of attachment formation during infancy. Therefore, in the present study we analyzed whether cortical responses to fearful and non-fearful faces at 7 months are associated with secure and insecure patterns of attachment after the first birthday. The ERP data have the potential to complement and extend the initial findings of differential attentional biases to fearful faces in securely vs. insecurely attached infants (Peltola et al., 2015) by indicating the early information processing stages that are most clearly associated with attachment (i.e., early 
perceptual processing reflected in the N290/P400 components vs. attentional processing indicated by the Nc component). Regarding the hypotheses, it should be noted that while a limited number of previous infant and child studies have observed attachment-related differences in both attention (Peltola et al., 2015) and perceptual sensitivity to facial emotions (Forslund, Kenward, Granqvist, Gredebäck, \& Brocki, 2017), there may not be a direct correspondence between the processes measured in these studies and the early neural responses measured with the ERP methodology (cf. Yrttiaho et al., 2014). Therefore, while we did hypothesize that insecure attachment would be associated with less pronounced cortical discrimination of fearful vs. non-fearful faces than secure attachment, our approach to whether such differences would be manifest in the perceptual (N290/P400) or attentional (Nc) ERP components was more exploratory.

Finally, in addition to the analyses comparing secure and insecure patterns of attachment, we conducted exploratory analyses comparing ERP responses between infants with and without attachment disorganization. Although our primary analyses were conducted with groups based on secure vs. insecure attachment due to the small number of infants in the different insecure subgroups, we considered that in light of previous findings and predictions derived from attachment theory it is nevertheless important to explore further whether attachment disorganization is associated with infants' ERP responses to fearful faces. It has been suggested that a core feature of disorganized attachment is defensive exclusion of threatening information that manifests in a tendency to orient attention away from stimuli signaling threat, possibly as an attempt to regulate overarousal (Bowlby, 1973, 1980; Dykas \& Cassidy, 2011; Reisz, Duschinsky, \& Siegel, 2018). While empirical findings linking disorganized types of adult attachment with suppressed attention to threat-related signals are scarce and mixed (Atkinson et al., 2009; Dewitte \& De Houwer, 2008), initial findings in infants (Peltola et al., 2015) indicated reduced processing of fearful faces in disorganized infants. Thus, we considered that it is important to explore this hypothesis further by analyzing infants' ERP responses in relation to attachment disorganization, while keeping in mind 
the limitations of such analyses due to highly unequal group sizes, and its post-hoc nature in the current case.

\section{Material and Methods}

\subsection{Participants}

The final sample consisted of 61 infants who provided ERP data at 7 months of age $(M=$ 213 days; $S D=2.96 ; 44 \%$ girls $)$ and attachment data at approximately 14 months of age $(M=415$ days; $S D=21.13$ ). Attachment was assessed from an additional 12 infants, who were excluded from the analyses due to excessive artefacts in the EEG data. The assessments were conducted as part of a longitudinal study consisting of laboratory assessments at 5, 7, 14, 24, and 48 months of age (Forssman et al., 2014; Peltola et al., 2015, 2013; Peltola, Yrttiaho, \& Leppänen, 2018; Yrttiaho et al., 2014). All infants were healthy, full-term ( $\geq 37$ weeks), and predominantly from urban, middle-class families of Caucasian ethnicity. Approval for the project was obtained from the Ethical Committee of Pirkanmaa Hospital District, and an informed written consent was obtained from the parent of each child.

\subsection{Measures}

2.2.1 ERPs to facial expressions. During the experiment, infants were sitting on the accompanying parent's lap at a distance of $60 \mathrm{~cm}$ from a 23 -inch monitor in a dimly lit room. EEG was recorded while the infants were presented with a task designed to assess attention to faces presented concurrently with peripheral distractor stimuli (Peltola et al., 2015; Yrttiaho et al., 2014). Each trial started with the presentation of a face or a control stimulus on the center of the screen for $1000 \mathrm{~ms}$, after which it was flanked for $3000 \mathrm{~ms}$ by a peripheral stimulus, presented $14^{\circ}$ equiprobably on the left or right. The central stimuli measured $15.4^{\circ} \times 10.8^{\circ}$ and they were neutral, happy, and fearful facial expressions posed by two female models and the face-shaped control stimuli were phase-scrambled images of both of the models' faces, preserving the outer contour of the face. The peripheral stimuli were black-and-white checkerboards or circle arrays, measuring 
$15.4^{\circ}$ and $4.3^{\circ}$ vertically and horizontally, respectively. Between each trial, a dynamic stimulus (a red circle dilating from $0.4^{\circ}$ to $4.3^{\circ}$ in a continuous fashion) was presented to attract the infant's attention to the center of the screen. The experimenter initiated each trial once the infant had fixated the dynamic stimulus for a minimum of $1000 \mathrm{~ms}$. The four facial stimuli from one model were presented during the first 24 trials (i.e., with 6 repetitions of each stimulus), after which the facial stimuli from the other model were presented during the following 24 trials, resulting in a total of 48 trials (i.e., maximum of 12 trials in each stimulus condition). The stimuli were presented in random order with the constraint that the peripheral stimulus was presented on the same side of the screen no more than four times in a row.

The experiment started with the application of a HydroCel 128-electrode sensor net (Electrical Geodesics Inc., Eugene, OR) on the infant's head and measurement of electrode impedances. The continuous EEG was recorded with a 250-Hz sampling rate and high-pass filtered at $0.3 \mathrm{~Hz}$. Offline, the Eegtool software (Kaatiala, Yrttiaho, Forssman, Perdue, \& Leppänen, 2014) was used to preprocess the EEG data for statistical analyses of the ERPs. First, based on video recordings temporally synced with the EEG signal, EEG data from trials containing gaze shifts away from the screen, blinks, facial and head movements, excessive body movements, or the parent or infant touching the electrodes during the first $1000 \mathrm{~ms}$ of the trial (i.e., when the face but not the distractor was displayed) were excluded from further processing. The following preprocessing steps were included 1) low-pass filtering of the EEG data at $30 \mathrm{~Hz}, 2$ ) segmentation to epochs spanning from $-100 \mathrm{~ms}$ before to $800 \mathrm{~ms}$ after the face stimulus onset in each stimulus condition, 3) detrending the epochs, 4) rejecting channels with impedance values above $200 \mathrm{k} \Omega$, 5) baselinecorrecting the EEG signal to the 100-ms prestimulus period, and 6) inspecting the epochs for residual artifacts. Electrodes in any epoch containing absolute amplitudes greater than $150 \mu \mathrm{V}$ were marked as bad and replaced with data interpolated from acceptable electrodes using spherical spline interpolation. However, if the number of bad EEG electrodes in an epoch was greater than 12 (i.e., 
about $10 \%$ of the 128 electrodes), the entire epoch was rejected. Finally, the EEG signal was rereferenced to the average from all electrodes.

The number of trials available for ERP analyses was lower than in typical infant ERP paradigms, mainly due to the 4000-ms duration of the trials in the infant attention task. Therefore, we set the limit for inclusion in the ERP analyses to 5 artefact-free trials per stimulus condition. To increase the power of the analyses and to address the specific goal of the study (i.e., investigate whether cortical responses specifically to fearful faces are associated with later attachment), the ERP responses to fearful faces were compared to a category of non-fearful faces consisting of both happy and neutral faces. As there were infants with less than 5 acceptable trials in either the happy or neutral stimulus condition, combining these stimuli into a single category increased the number of infants included in the analyses from 55 to 61 . Using a combined category of non-fearful faces is also justified by previous analyses which showed no differences in ERP responses to happy and neutral faces in this dataset (Yrttiaho et al., 2014). An average of $8.34(S D=1.95)$ fearful and 16.43 $(S D=4.17)$ non-fearful epochs were retained for the ERP analyses. The ERPs to the control stimuli were clearly distinctive from those to the real face stimuli, possibly owing to their oddball status in relation to the other stimuli (Yrttiaho et al., 2014), and are therefore not reported here.

ERPs were analyzed from posterior (N290 and P400) and central (Nc) electrode sites. The N290 and P400 components were analyzed from posterior electrode sets covering areas of the left [electrodes 58(T6), 59, 64, 65, 66, 70(O1)] and right-hemisphere [electrodes 83(O2), 84, 90, 91, 95, 96(T6)] occipitotemporal cortex. The N290 was quantified from these electrodes as the mean amplitude between 250 and $350 \mathrm{~ms}$, and the P400 as the mean amplitude between 350 and $550 \mathrm{~ms}$ after stimulus onset. Visual inspection of the grand average ERP graphs showed that with the average-referenced data, the amplitudes of the Nc component were small and the waveform not clearly visible. Therefore, for the Nc analyses, the data were re-referenced to the mastoid electrodes (57 and 100), resulting in a much more pronounced Nc waveform. The Nc was then quantified as 
the mean amplitude between 350 and $600 \mathrm{~ms}$ after stimulus onset on the left [electrodes 29, 30, 35, 36(C3), 37, 42] and right-hemisphere [electrodes 87, 93, 104(C4), 105, 110, 111]) mid-central area. In all analyses, ERP amplitudes were averaged across both hemispheres, as there were no a priori reasons to expect meaningful hemisphere-specific effects, and the potential three-way interactions including hemisphere would have been underpowered.

2.2.2 Infant-mother attachment. Infants and mothers were observed in the Strange Situation Procedure (Ainsworth et al., 1978). The SSP consisted of seven 3-minute episodes, including two separations from and two reunions with the mother, and interaction with a female stranger. Infants' attachment behaviors were coded from videotapes according to the organized attachment scales by Ainsworth et al. (1978) and the Main and Solomon (1990) coding system for assessing attachment disorganization. On the basis of ratings on 7-point scales assessing infants' proximity seeking, contact maintenance, resistance, and avoidance during the two reunion episodes, infants were first classified as secure (B), insecure-avoidant (A), or insecure-resistant (C). Signs of attachment disorganization (D) during each episode when the mother was present were then rated using a scale from 1 (no signs of disorganization) to 9 (strong signs of disorganization), with scores higher than 5 receiving a disorganized classification. Intercoder agreement was calculated from $18 \%$ of the sample by two coders (authors 1 and 2). For the 4-way ABCD classifications, presence vs. absence of secure attachment (i.e., B vs. non-B), and the presence vs. absence of attachment disorganization (i.e., D vs. non-D), intercoder agreement was $92 \%$ in each comparison $(\kappa=.88, \kappa=$ .85 , and $\kappa=.81$, respectively). Agreement on the continuous D scores (intraclass correlation) was $I C C=.91$. For the remaining sample, the $\mathrm{ABCD}$ classifications were based on a consensus between the first author and expert coders from Leiden Center for Child and Family Studies. In the full sample of 73 infants, $63 \%$ of the infants received a secure (B) classification, $14 \%$ an insecureavoidant (A), $8 \%$ an insecure-resistant (C), and $15 \%$ a disorganized (D) classification. Within the sample of 61 infants having sufficient ERP data at 7 months, the distribution was: $\mathrm{B}=67 \%, \mathrm{~A}=$ 
$13 \%, \mathrm{C}=7 \%$, and $\mathrm{D}=13 \%$. The primary statistical analyses were conducted using a secure $(\mathrm{B} ; n=$ 41) vs. insecure grouping (A, C, D; $n=20$ ). In addition, exploratory analyses were conducted to compare infants with a D classification $(n=8)$ to B infants $(n=41)$ as well as to infants with an organized attachment classification (A, B, C; $n=53)$.

\section{Results}

In the primary analyses, the ERP data were analyzed separately for each component of interest with a 2 × 2 analysis of variance (ANOVA) with Emotion (fearful, non-fearful) as a withinsubjects factor and Attachment (secure, insecure) as a between-subjects factor. Initially, the potential influence of maternal sensitivity (assessed at 7 months during a free-play assessment with the Emotional Availability Scales; Biringen, 2008) was taken into account by adding it as a covariate in the analysis. However, as maternal sensitivity did not significantly interact with the variables included in the ANOVA, it was excluded from the final analyses.

\section{$3.1 \mathrm{~N} 290$}

For the N290 amplitudes, the 2-way Emotion x Attachment interaction was significant, $F(1$, 59) $=9.95, p=.003, n_{p}^{2}=.14$, indicating a medium effect size according to conventional criteria (Cohen, 1988). To break down this interaction, the effects were inspected separately for the attachment groups. Securely attached infants showed a significant and medium-sized main effect of Emotion, $F(1,40)=9.66, p=.003, n_{p}^{2}=.20$. The $\mathrm{N} 290$ amplitudes were thus more positive to fearful $(M=4.25 \mu \mathrm{V}, S E=2.16)$ than to non-fearful faces $(M=-1.48 \mu \mathrm{V}, S E=1.52)$ in securely attached infants (Figure 1). In insecurely attached infants, no significant main effect of Emotion was observed, $F(1,19)=2.57, p=.13, n_{p}^{2}=.12$.

Figure 1 about here

\subsection{P400}


For the P400 amplitudes, the Emotion x Attachment interaction was not significant at the nominal alpha level, $F(1,59)=3.35, p=.07, n_{p}^{2}=.05 .^{1}$

\section{$3.3 \mathrm{Nc}$}

In the analysis of mean amplitudes during the Nc time window at prefrontally located channels, the Emotion $\mathrm{x}$ Attachment interaction was not significant, $F(1,59)=2.72, p=.10, n_{p}^{2}=$ .04 .

\subsection{Associations Between ERP Responses and Attachment Disorganization}

As an exploratory analysis, the ANOVAs reported above were first repeated with attachment disorganization vs. security (D, B) as a between-subjects factor. Similarly as above, the analysis of N290 amplitudes showed a significant Emotion $\mathrm{x}$ Disorganization interaction, $F(1,47)=$ $8.60, p=.005, n_{p}^{2}=.16$, which emerged from more pronounced ERP differentiation of the emotion conditions in the securely attached infants, $F(1,40)=9.66, p=.003, n_{p}^{2}=.20$, as compared to infants with disorganized attachment, $F(1,7)=2.39, p=.17, n_{p}^{2}=.25$. For the P400 and Nc components, no Emotion x Disorganization interactions were observed, $F(1,47)=2.52, p=.12, n_{p}^{2}$ $=.05$, and $F(1,47)=1.19, p=.28, n_{p}^{2}=.03$, respectively. Second, using a disorganized vs. organized grouping resulted in an identical pattern of a significant interaction for the $\mathrm{N} 290, F(1,59)$ $=5.39, p=.02, n_{p}^{2}=.08$, and non-significant interactions for the $\mathrm{P} 400, F(1,59)=1.77, p=.19, n_{p}^{2}$ $=.03$, and Nc components, $F(1,59)=0.83, p=.37, n_{p}^{2}=.01$.

\section{Discussion}

The present results suggest that attachment security at 14 months of age is related to cortical discrimination of fearful from non-fearful faces at 7 months of age. Infants who were later classified as insecurely attached (i.e., avoidant, resistant, or disorganized) did not display clear differences in ERPs to fearful vs. non-fearful faces in the N290, P400, or Nc ERP components. Securely attached infants displayed robust ERP discrimination of fearful vs. non-fearful faces in the amplitudes of the $\mathrm{N} 290$ response, with a medium effect size according to conventional criteria 
(Cohen, 1988). No attachment-related effects were observed in the Nc amplitudes, which are related to attention allocation. An exploratory analysis comparing infants with disorganized vs. secure and disorganized vs. organized attachment classifications indicated a similar group difference in the N290 responses.

Together with previous findings showing associations between attachment and attentional biases to fearful faces (Peltola et al., 2015), the present results converge to suggest that patterns of secure and insecure infant attachment are related to early-emerging differences in perceiving and attending to facial emotions. While the attentional biases and ERP discrimination of fearful faces in securely attached infants replicate age-typical findings from other studies using non-selected samples (Jessen \& Grossmann, 2015; Leppänen et al., 2007; Peltola, Leppänen, \& Hietanen, 2011; Peltola et al., 2008), insecure and disorganized infant attachment appears to be characterized by reduced age-typical cortical and attentional responses to fearful faces. It is important to note that in previous analyses of the current dataset (Yrttiaho et al., 2014), no clear correlations were observed between ERP amplitudes and eye-tracking based attention biases to faces. Therefore, the associations of eye-tracking based attention biases (Peltola et al., 2015) and ERPs with attachment security may be independent and ERPs cannot definitely answer whether the attachment-related effects on processing emotional faces are primarily driven by early perceptual processes or later attentional processes. ERP responses may specifically index the early automatic stages of face processing, which may or may not contribute to the more voluntary attention orienting between faces and competing stimuli.

As a potential precursor to the adult N170 component, the differentiation of emotional expressions particularly in the N290 component is suggested to reflect the activity of posterior temporal face-sensitive cortical areas located in the fusiform gyrus and the superior temporal sulcus (de Haan et al., 2003; Guy et al., 2016; Leppänen et al., 2007), while the P400 may recruit more broadly distributed cortical sources (Guy et al., 2016). Modulation of the Nc component, on the 
other hand, has been characterized to represent the degree of prefrontally controlled attention allocation to different stimuli (Courchesne, Ganz, \& Norcia, 1981; Reynolds \& Richards, 2005). Therefore, as attachment-related modulation by fearful vs. non-fearful faces was observed in the N290 responses but not in the amplitudes of the Nc component, the present results may indicate that the differences between securely and insecurely attached or disorganized infants in attending to fearful faces may emerge from early perceptual processes associated with the structural analysis of different facial features rather than differences in the automatic capture of attention by fearful faces. However, this suggestion should be considered tentative and it is critical that the results are replicated in larger samples with a larger number of trials included in the ERP calculations in order to make more reliable conclusions about the underlying information processing mechanisms and to verify whether the absence of ERP differences to fearful vs. non-fearful faces in insecurely attached and disorganized infants is a replicable finding. It should also be noted that the functional properties of the different infant ERP components are not unequivocal, as the N290 and P400 are also sensitive to the degree of attention and the $\mathrm{P} 400$, in particular, may in fact represent a polarity reversal of the more frontal Nc response (Guy et al., 2016), thus not necessarily purely reflecting activity of face-sensitive visual cortical areas.

The developmental origins of the ERP differences between the attachment groups at 7 months are unclear. One possibility is that elements of face-to-face interaction between caregivers and infants shape infants' developing sensitivity to variations in facial emotion signaling. Although the rather global assessment of maternal sensitivity was not associated with the ERP responses in the present study, more fine-grained interaction analyses might reveal subtle markers of early faceto-face interaction that become associated with infants' perceptual and attentional responses to facial emotions. For example, factors that are associated with the development of insecure and particularly disorganized attachment, such as inconsistent or deficient parental mirroring of infant emotions, expression of conflicting facial emotions, or insufficient exposure to emotional 
expressions due to parental withdrawal from interaction with the infant (Beebe et al., 2010; DeOliveira, Bailey, Moran, \& Pederson, 2004; Lyons-Ruth, Yellin, Melnick, \& Atwood, 2005) could shape the way infants learn to discriminate and attend to emotions on others' faces (cf. Rayson et al., 2017). From this perspective, infants' cortical sensitivity to facial emotions could be considered as a correlate of the caregiving environment and an early manifestation of more general attachment-related biases in social information processing.

Alternatively, sensitivity to facial emotions may reflect an inherent characteristic of the infant, and could thus be relatively independently associated with later patterns of attachment. Preliminary findings indicate that individual differences in infants' sensitivity to fearful faces are associated with genetic polymorphisms affecting major neurotransmitter systems (Forssman et al., 2014; Grossmann et al., 2011; Leppänen et al., 2011). Furthermore, evidence of interaction between genetic and maternal characteristics in predicting infants' responses to fearful faces (Forssman et al., 2014) also suggests that differential susceptibility to the influence of the caregiving environment (Ellis, Boyce, Belsky, Bakermans-Kranenburg, \& van IJzendoorn, 2011) may play a role in the development of emotion processing in infancy. Teasing apart these distinct and potentially interactive influences on attachment development will require larger longitudinal studies incorporating more focal assessments of caregiver-infant interaction patterns and their relations to developmental changes in infants' sensitivity to facial emotions.

Patterns of attachment may be associated with alterations in emotion processing that extend far beyond infancy and have critical implications for social development. The small number of studies investigating attachment and emotion processing in older children have shown that insecure and particularly disorganized attachment is associated with poorer identification of facial expressions (Forslund et al., 2017; Steele, Steele, \& Croft, 2008). Similar results have been observed in adolescents (Escobar et al., 2013) and adults (Fraley, Niedenthal, Marks, Brumbaugh, \& Vicary, 2006). Developmentally, lower sensitivity and attentiveness to facial expressions in 
insecurely attached and disorganized infants could limit their learning opportunities during social interactions and adaptive regulation of behavior according to subtle emotional facial signals communicated by peers and caregivers. Consequently, such processes may contribute to observations of poorer social competence with peers (Groh et al., 2014) and higher rate of externalizing behavioral problems (Fearon, Bakermans-Kranenburg, van IJzendoorn, Lapsley, \& Roisman, 2010) in insecurely attached and disorganized children.

The generalizability of the present findings is limited. First, as fearful faces were the only negative emotion category, it is unclear whether the attachment-related ERP modulation is specific to the signal conveyed by fearful faces, or whether it reflects a more general response to negative emotional expressions. Fearful faces were selected as the negative emotion stimulus in this study because in previous infant research most robust attentional and ERP effects have been observed in response to fearful faces, while similar responses to other negative faces such as angry faces are less apparent (Grossmann, Striano, \& Friederici, 2006; Soken \& Pick, 1999). Second, the number of trials available for ERP analyses was smaller than is commonly recommended in infant ERP research (Hoehl \& Wahl, 2012), which may have contributed to the lack of clear effects in the Nc component (cf. Stets \& Reid, 2011). It should be noted, however, that in previous analyses of the present ERP data (Yrttiaho et al., 2014), the ERP differences between fearful vs. non-fearful faces in the N290 component remained unchanged when using more stringent inclusion criteria (i.e., a minimum of 10 accepted trials), indicating that the effects observed in the current data were not driven by the number of trials included in the analyses.

The sample size in the present study was too small to permit separate analyses for the insecure attachment subgroups. It will be important to replicate the study with a larger sample of infants to determine whether or which of the subgroups show theoretically meaningful differences in cortical and attentional sensitivity to facial emotions. For example, it would be important to test whether avoidant and resistant patterns of attachment are associated with down-regulated and 
hypervigilant patterns of emotion processing, respectively, as suggested by studies investigating the associations between adult attachment style and facial emotion processing (Fraley et al., 2006; Niedenthal, Brauer, Robin, Innes-Ker, \& Innes-Ker, 2002). Similarly, while the analyses comparing infants with disorganized vs. secure and disorganized vs. organized attachment suggested a similar group difference in the N290 responses as the analysis comparing securely and insecurely attached infants, these analyses were based on highly unequal group sizes and, therefore, should be considered as purely exploratory. In the future, it will be important to obtain infant samples with larger representation of attachment disorganization symptoms to enable further investigation of key hypotheses relating attachment disorganization to emotion processing, such as whether disorganization is associated with defensive exclusion and suppression of attention in response to threat-related cues (e.g., Reisz et al., 2018). Demonstrating such associations in early development could provide important clues for understanding the mechanisms by which attachment disorganization increases the likelihood of externalizing and peer problems (Fearon et al., 2010; Groh et al., 2014; Peltola et al., 2015). It will also be highly interesting to investigate in greater detail how exactly do avoidant and disorganized infant attachments differ with respect to processing threat-related signals. Both avoidance and disorganization may be linked to habitual suppression of attention to threat-related signals, but in disorganized infants this pattern may be more pervasive, which may block access to the attachment behavioral system and thereby hamper organized attachment behavior upon stress (Bowlby, 1973, 1980; Reisz et al., 2018). One possibility to address this proposition could be to measure infants' processing of threat-related signals before as well as during a stress-eliciting manipulation.

To conclude, the present results add to previous findings (Peltola et al., 2015) by suggesting that patterns of secure and insecure infant attachment at one year of age are related to cortical discrimination of fearful and non-fearful faces, with securely, but not insecurely, attached infants showing age-typical ERP differentiation of fearful and non-fearful faces at 7 months of age. In the 
future, larger studies integrating ERP methodology to the study of infant attachment development will provide fruitful opportunities to identify the components of emotional information processing which are most sensitive to variations in infant attachment security, and also allow testing with greater precision whether disorganized and insecure patterns of attachment are associated with subgroup-specific neural responses to emotional and particularly threat-related facial signals. The investigation of emotion processing and its neural bases in developmental populations will advance attachment theory and research by complementing research on theoretical concepts central to attachment formation, such as internal working models, with directly measurable core processes of social interaction, such as the decoding of emotional signals from faces. 


\section{Notes}

1. Given the marginal Emotion x Attachment interaction in the P400 amplitudes and the exploratory nature of the study, we were inclined to explore simple main effects within the attachment groups. In securely attached infants, a main effect of emotion was observed, $F(1,40)=4.10, p=.05, n_{p}^{2}=$ .09 , indicating a small effect of more positive P400 amplitudes to fearful $(M=18.88 \mu \mathrm{V}, S E=1.89)$ than to non-fearful faces $(M=16.00 \mu \mathrm{V}, S E=1.67)$ in securely attached infants (Figure 1$)$. In insecurely attached infants, no significant main effect of Emotion was observed, $F(1,19)=0.65, p$ $=.43, n_{p}^{2}=.03$. 


\section{Declaration of interest}

The authors declare no conflicts of interest. 


\section{References}

Ainsworth, M. D. S., Blehar, M. C., Waters, E., \& Wall, S. (1978). Patterns of attachment: A psychological study of the strange situation. Hillsdale, NJ: Erlbaum.

Atkinson, L., Leung, E., Goldberg, S., Benoit, D., Poulton, L., Myhal, N., ... Kerr, S. (2009). Attachment and selective attention: Disorganization and emotional Stroop reaction time. Development and Psychopathology, 21(1), 99-126. https://doi.org/10.1017/S0954579409000078

Beebe, B., Jaffe, J., Markese, S., Buck, K., Chen, H., Cohen, P., ... Feldstein, S. (2010). The origins of 12-month attachment: A microanalysis of 4-month mother-infant interaction. Attachment \& Human Development, 12(1-2), 3-141. https://doi.org/10.1080/14616730903338985

Biringen, Z. (2008). Emotional Availability (EA) Scales (4th ed.). Retrieved from http://www.emotionalavailability.com.

Bowlby, J. (1973). Attachment and loss: Vol. 2. Separation. New York, NY: Basic Books.

Bowlby, J. (1980). Attachment and loss: Vol. 3. Loss. New York, NY: Basic Books.

Cassidy, J., Ehrlich, K. B., \& Sherman, L. J. (2013). Child-parent attachment and response to threat: A move from the level of representation. In M. Mikulincer \& P. R. Shaver (Eds.), Nature and development of social connections: From brain to group. (pp. 125-144). Washington D.C.: American Psychological Association. https://doi.org/10.1037/14250-008

Cohen, J. (1988). Statistical Power Analysis for the Behavioral Sciences (2nd ed.). Lawrence Erlbaum Associates.

Courchesne, E., Ganz, L., \& Norcia, A. M. (1981). Event-related brain potentials to human faces in infants. Child Development, 52(3), 804-811.

de Haan, M., Johnson, M. H., \& Halit, H. (2003). Development of face-sensitive event-related potentials during infancy: A review. International Journal of Psychophysiology, 51(1), 45. https://doi.org/10.1016/S0167-8760(03)00152-1 
De Wolff, M. S., \& van IJzendoorn, M. H. (1997). Sensitivity and attachment: A meta-analysis on parental antecedents of infant attachment. Child Development, 68(4), 571-591. https://doi.org/10.1111/j.1467-8624.1997.tb04218.x

DeOliveira, C. A., Bailey, H. N., Moran, G., \& Pederson, D. R. (2004). Emotion socialization as a framework for understanding the development of disorganized attachment. Social Development, 13(3), 437-467. https://doi.org/10.1111/j.1467-9507.2004.00276.x

Dewitte, M., \& De Houwer, J. (2008). Adult attachment and attention to positive and negative emotional face expressions. Journal of Research in Personality, 42(2), 498-505. https://doi.org/10.1016/j.jrp.2007.07.010

Dykas, M. J., \& Cassidy, J. (2011). Attachment and the processing of social information across the life span: Theory and evidence. Psychological Bulletin, 137(1), 19-46. https://doi.org/10.1037/a0021367

Ellis, B. J., Boyce, W. T., Belsky, J., Bakermans-Kranenburg, M. J., \& van IJzendoorn, M. H. (2011). Differential susceptibility to the environment: An evolutionary--neurodevelopmental theory. Development and Psychopathology, 23(1), 7-28. https://doi.org/10.1017/S0954579410000611

Escobar, M. J., Rivera-Rei, A., Decety, J., Huepe, D., Cardona, J. F., Canales-Johnson, A., ... Ibañez, A. (2013). Attachment patterns trigger differential neural signature of emotional processing in adolescents. PLoS ONE, 8(8), e70247. https://doi.org/10.1371/journal.pone.0070247

Fearon, R. P., Bakermans-Kranenburg, M. J., van IJzendoorn, M. H., Lapsley, A.-M. M., \& Roisman, G. I. (2010). The significance of insecure attachment and disorganization in the development of children's externalizing behavior: A meta-analytic study. Child Development, 81(2), 435-456. https://doi.org/10.1111/j.1467-8624.2009.01405.x

Forslund, T., Kenward, B., Granqvist, P., Gredebäck, G., \& Brocki, K. C. (2017). Diminished 
ability to identify facial emotional expressions in children with disorganized attachment representations. Developmental Science, 20(6), e12465. https://doi.org/10.1111/desc.12465

Forssman, L., Peltola, M. J., Yrttiaho, S., Puura, K., Mononen, N., Lehtimäki, T., \& Leppänen, J. M. (2014). Regulatory variant of the TPH2 gene and early life stress are associated with heightened attention to social signals of fear in infants. Journal of Child Psychology and Psychiatry, 55(7), 793-801. https://doi.org/10.1111/jcpp.12181

Fraley, C. R., Niedenthal, P. M., Marks, M., Brumbaugh, C., \& Vicary, A. (2006). Adult attachment and the perception of emotional expressions: Probing the hyperactivating strategies underlying anxious attachment. Journal of Personality, 74(4), 1163-1190. https://doi.org/10.1111/j.14676494.2006.00406.x

Groh, A. M., Fearon, R. P., Bakermans-Kranenburg, M. J., van IJzendoorn, M. H., Steele, R. D., \& Roisman, G. I. (2014). The significance of attachment security for children's social competence with peers: A meta-analytic study. Attachment \& Human Development, 16(2), 103-136. https://doi.org/10.1080/14616734.2014.883636

Grossmann, T., Johnson, M. H., Vaish, A., Hughes, D., Quinque, D., Stoneking, M., \& Friederici, A. D. (2011). Genetic and neural dissociation of individual responses to emotional expressions in human infants. Developmental Cognitive Neuroscience, 1(1), 57-66. https://doi.org/10.1016/j.den.2010.07.001

Grossmann, T., Striano, T., \& Friederici, A. D. (2006). Developmental changes in infants’ processing of happy and angry facial expressions: A neurobehavioral study. Brain and Cognition, 64(1), 30-41. https://doi.org/10.1016/j.bandc.2006.10.002

Guy, M. W., Zieber, N., \& Richards, J. E. (2016). The cortical development of specialized face processing in infancy. Child Development, 87(5), 1581-1600. https://doi.org/10.1111/cdev.12543

Hoehl, S., \& Striano, T. (2010). The development of emotional face and eye gaze processing. 
Developmental Science, 13(6), 813-825. https://doi.org/10.1111/j.1467-7687.2009.00944.x

Hoehl, S., \& Wahl, S. (2012). Recording infant ERP data for cognitive research. Developmental Neuropsychology, 37(3), 187-209. https://doi.org/10.1080/87565641.2011.627958

Jessen, S., \& Grossmann, T. (2015). Neural signatures of conscious and unconscious emotional face processing in human infants. Cortex, 64, 260-270. https://doi.org/10.1016/j.cortex.2014.11.007

Kaatiala, J., Yrttiaho, S., Forssman, L., Perdue, K., \& Leppänen, J. M. (2014). A graphical user interface for infant ERP analysis. Behavior Research Methods, 46(3), 745-757. https://doi.org/10.3758/s13428-013-0404-4

Kim, S., Fonagy, P., Allen, J., Martinez, S., Iyengar, U., \& Strathearn, L. (2014). Mothers who are securely attached in pregnancy show more attuned infant mirroring 7 months postpartum. Infant Behavior and Development, 37(4), 491-504. https://doi.org/10.1016/j.infbeh.2014.06.002

Leppänen, J. M., Moulson, M. C., Vogel-farley, V. K., \& Nelson, C. A. (2007). An ERP study of emotional face processing in the adult and infant brain. Child Development, 78(1), 232-245. https://doi.org/10.1111/j.1467-8624.2007.00994.x

Leppänen, J. M., Peltola, M. J., Puura, K., Mäntymaa, M., Mononen, N., \& Lehtimäki, T. (2011). Serotonin and early cognitive development: Variation in the tryptophan hydroxylase 2 gene is associated with visual attention in 7-month-old infants. Journal of Child Psychology and Psychiatry, 52(11), 1144-1152. https://doi.org/10.1111/j.1469-7610.2011.02391.x

Lyons-Ruth, K., Yellin, C., Melnick, S., \& Atwood, G. (2005). Expanding the concept of unresolved mental states: Hostile/helpless states of mind on the Adult Attachment Interview are associated with disrupted mother-infant communication and infant disorganization. Development and Psychopathology, 17(1), 1-23. https://doi.org/10.1017/S0954579405050017 Main, M., \& Solomon, J. (1990). Procedures for identifying infants as disorganized/disoriented 
during the Ainsworth Strange Situation. In M. T. Greenberg, D. Cicchetti, \& E. M. Cummings (Eds.), Attachment in the preschool years: Theory, research and intervention (pp. 121-160). Chicago, IL: University of Chicago Press.

Nelson, C. A., \& de Haan, M. (1996). Neural correlates of infants' visual responsiveness to facial expressions of emotion. Developmental Psychobiology, 29(7), 577-595. https://doi.org/10.1002/(SICI)1098-2302(199611)29:7<577::AID-DEV3>3.0.CO;2-R

Niedenthal, P. M., Brauer, M., Robin, L., Innes-Ker, A. H., \& Innes-Ker, Å. H. (2002). Adult attachment and the perception of facial expression of emotion. Journal of Personality and Social Psychology, 82(3), 419-433. https://doi.org/10.1037/0022-3514.82.3.419

Peltola, M. J., Forssman, L., Puura, K., van IJzendoorn, M. H., \& Leppänen, J. M. (2015). Attention to faces expressing negative emotion at 7 months predicts attachment security at 14 months. Child Development, 86(5), 1321-1332. https://doi.org/10.1111/cdev.12380

Peltola, M. J., Hietanen, J. K., Forssman, L., \& Leppänen, J. M. (2013). The emergence and stability of the attentional bias to fearful faces in infancy. Infancy, 18(6), 905-926. https://doi.org/10.1111/infa.12013

Peltola, M. J., Leppänen, J. M., \& Hietanen, J. K. (2011). Enhanced cardiac and attentional responding to fearful faces in 7-month-old infants. Psychophysiology, 48(9), 1291-1298. https://doi.org/10.1111/j.1469-8986.2011.01188.x

Peltola, M. J., Leppänen, J. M., Mäki, S., \& Hietanen, J. K. (2009). Emergence of enhanced attention to fearful faces between 5 and 7 months of age. Social Cognitive and Affective Neuroscience, 4(2), 134-42. https://doi.org/10.1093/scan/nsn046

Peltola, M. J., Leppänen, J. M., Palokangas, T., \& Hietanen, J. K. (2008). Fearful faces modulate looking duration and attention disengagement in 7-month-old infants. Developmental Science, 11(1), 60-68. https://doi.org/10.1111/j.1467-7687.2007.00659.x

Peltola, M. J., Yrttiaho, S., \& Leppänen, J. M. (2018). Infants' attention bias to faces as an early 
marker of social development. Developmental Science, e12687.

https://doi.org/10.1111/desc.12687

Rayson, H., Bonaiuto, J. J., Ferrari, P. F., \& Murray, L. (2017). Early maternal mirroring predicts infant motor system activation during facial expression observation. Scientific Reports, 7, 11738. https://doi.org/10.1038/s41598-017-12097-w

Reisz, S., Duschinsky, R., \& Siegel, D. J. (2018). Disorganized attachment and defense: Exploring John Bowlby's unpublished reflections. Attachment and Human Development, 20(2), 107-134. https://doi.org/10.1080/14616734.2017.1380055

Reynolds, G. D., \& Richards, J. E. (2005). Familiarization, attention, and recognition memory in infancy: An event-related potential and cortical source localization study. Developmental Psychology, 41(4), 598-615. https://doi.org/10.1037/0012-1649.41.4.598

Sadeh, B., Podlipsky, I., Zhdanov, A., \& Yovel, G. (2010). Event-related potential and functional MRI measures of face-selectivity are highly correlated: A simultaneous ERP-fMRI investigation. Human Brain Mapping, 31(10), 1490-1501. https://doi.org/10.1002/hbm.20952

Soken, N. H., \& Pick, A. D. (1999). Infants' perception of dynamic affective expressions: Do infants distinguish specific expressions? Child Development, 70(6), 1275-1282. https://doi.org/10.1111/1467-8624.00093

Steele, H., Steele, M., \& Croft, C. (2008). Early attachment predicts emotion recognition at 6 and 11 years old. Attachment \& Human Development, 10(4), 379-393. https://doi.org/10.1080/14616730802461409

Stets, M., \& Reid, V. M. (2011). Infant ERP amplitudes change over the course of an experimental session: Implications for cognitive processes and methodology. Brain and Development, 33(7), 558-568. https://doi.org/10.1016/j.braindev.2010.10.008

Verhage, M. L., Schuengel, C., Madigan, S., Fearon, R. M. P., Oosterman, M., Cassibba, R., ... Van Ijzendoorn, M. H. (2016). Narrowing the transmission gap: A synthesis of three decades 
of research on intergenerational transmission of attachment. Psychological Bulletin, 142(4), 337-366. https://doi.org/10.1037/bul0000038

Yrttiaho, S., Forssman, L., Kaatiala, J., \& Leppänen, J. M. (2014). Developmental precursors of social brain networks: The emergence of attentional and cortical sensitivity to facial expressions in 5 to 7 months old infants. PLOS ONE, 9(6):e100811. https://doi.org/10.1371/journal.pone.0100811 


\section{Figure Captions}

Figure 1. Event-related potentials to fearful (red) and non-fearful (blue) faces in the occipitotemporal electrode sets in securely attached (above) and insecurely attached infants (below). The thin lines depict bootstrapped 95\% confidence intervals based on the resampling distributions (1000x) of the ERP waveforms using all accepted trials (see Yrttiaho et al., 2014, for details). 

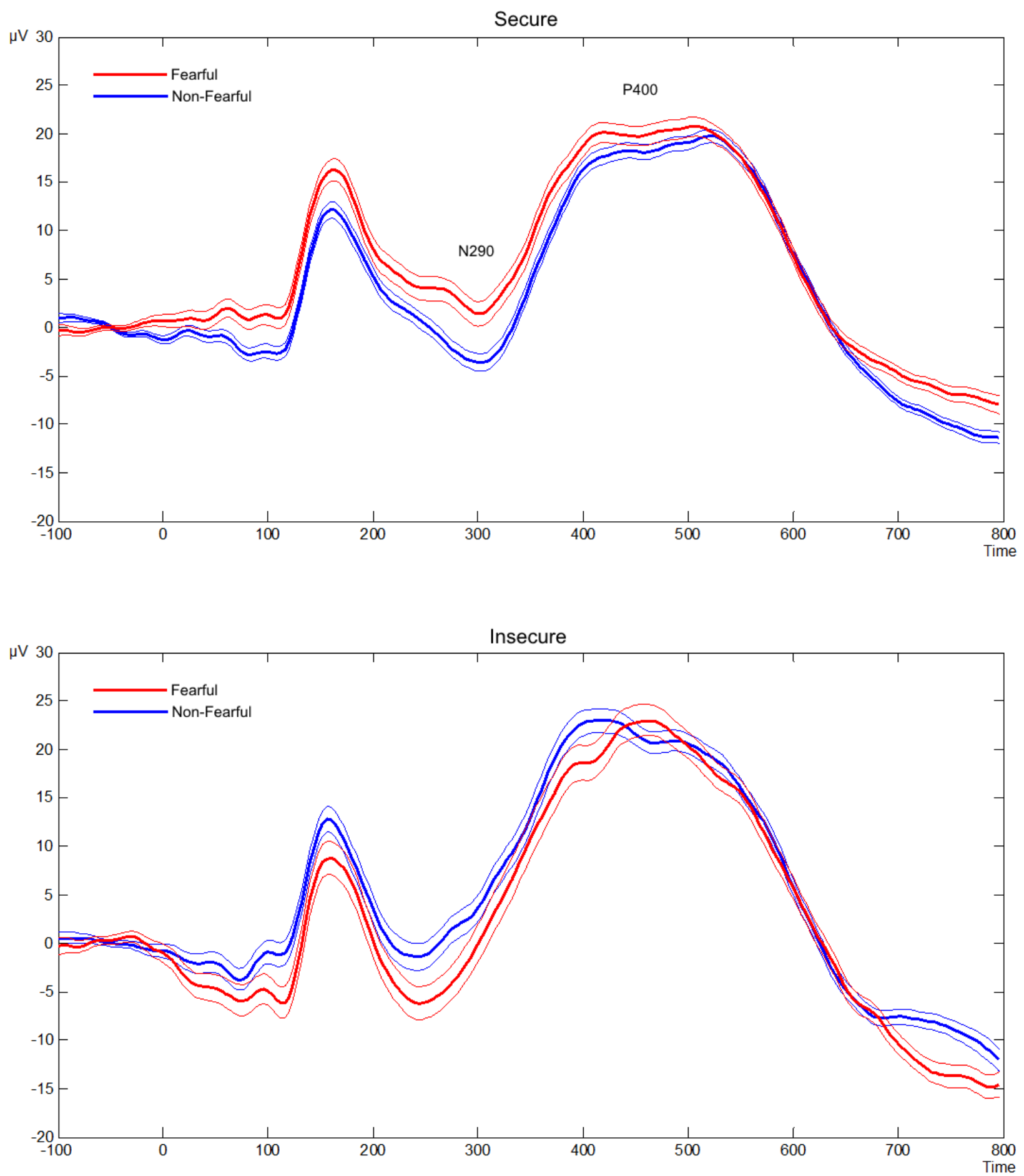

Figure 1. 